\title{
Mechanism of UVA Degradation of Synthetic Eumelanin
}

Weiyao Li, ${ }^{1,}+$ Zhao Wang, ${ }^{2,3},{ }^{\dagger}$ Ming Xiao, ${ }^{1,}+$ Toshikazu Miyoshi, ${ }^{1}$ Xiaozhou Yang, ${ }^{1}$ Ziying Hu, ${ }^{3}$ Cheng Liu, ${ }^{1}$ Steven S.C. Chuang, ${ }^{1}$ Matthew D. Shawkey, ${ }^{,}$* Nathan C. Gianneschi, ${ }^{2,3,4,6, *}$ and Ali Dhinojwala ${ }^{1, *}$

${ }^{1}$ Department of Polymer Science, The University of Akron, Akron, Ohio 44325, United States

${ }^{2}$ Department of Chemistry \& Biochemistry, University of California, San Diego, La Jolla, California 92093, United States

${ }^{3}$ Department of Chemistry, Northwestern University, Evanston, Illinois 60208, United States.

${ }^{4}$ Department of Materials Science \& Engineering, Northwestern University, Evanston, Illinois 60208, United States.

${ }^{5}$ Department of Biology, Evolution and Optics of Nanostructures Group, Ledeganckstraat 35, University of Ghent, Ghent 9ooo, Belgium

${ }^{6}$ Department of Biomedical Engineering, Northwestern University, Evanston, Illinois 60208, United States.

${ }^{\dagger}$ These authors contributed equally to this work.

\section{Supporting Information}

\section{Calculation for UVA dose}

UVA dose $(\mathrm{J} / \mathrm{g})=$ UVA intensity $\left(\mathrm{W} / \mathrm{cm}^{2}\right) \times$ Exposed area $\left(\mathrm{cm}^{2}\right) \times$ Exposure time $(\mathrm{s}) /$ Eumelanin mass $(\mathrm{g})$

UVA intensity $=2.43 \pm 0.02\left(\mathrm{~W} / \mathrm{cm}^{2}\right)$

For Transmission FTIR: Area of top-circle side of $\mathrm{KBr}$ pellet $=\pi / 4 \times$ diameter $(1.25 \pm 0.01)^{2}=1.23 \pm 0.02 \mathrm{~cm}^{2} ;$ exposure time $=30 \mathrm{~min} ;$ eumelanin mass $=0.20 \pm 0.05$ mg. UVA dose $\approx(2.7 \pm 0.7) \times 10^{7} \mathrm{~J} / \mathrm{g}$.

For ssNMR and DRIFTS on unlabeled sample: Area of spreaded eumelanin powder $=$ $1.8 \pm 0.4 \mathrm{~cm}^{2} ;$ exposure time $=2 \mathrm{hr}$; eumelanin mass $=50 \pm 5 \mathrm{mg}$. UVA dose $\approx(6.3 \pm 2.1)$ $\times 10^{5} \mathrm{~J} / \mathrm{g}$.

For ssNMR on ${ }^{13} \mathrm{C}$ labeled PDA: Area of spreaded eumelanin powder $=1.8 \pm 0.4 \mathrm{~cm}^{2}$; exposure time $=2 \mathrm{hr}$; eumelanin mass $=5.3 \pm 0.1 \mathrm{mg}$. UVA dose $\approx(5.9 \pm 1.5) \times 10^{6} \mathrm{~J} / \mathrm{g}$. 


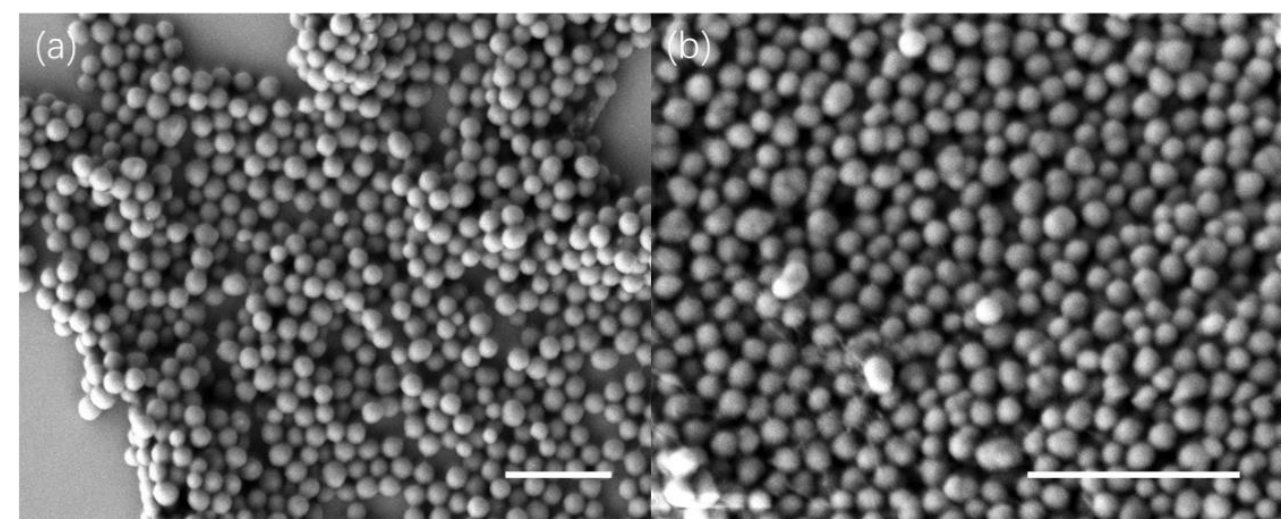

Figure S1. SEM images of nanoparticles with uniform size (a) PDA spherical nanoparticles. (b) PDOPA spherical nanoparticles. Scale bar: $1 \mu \mathrm{m}$.

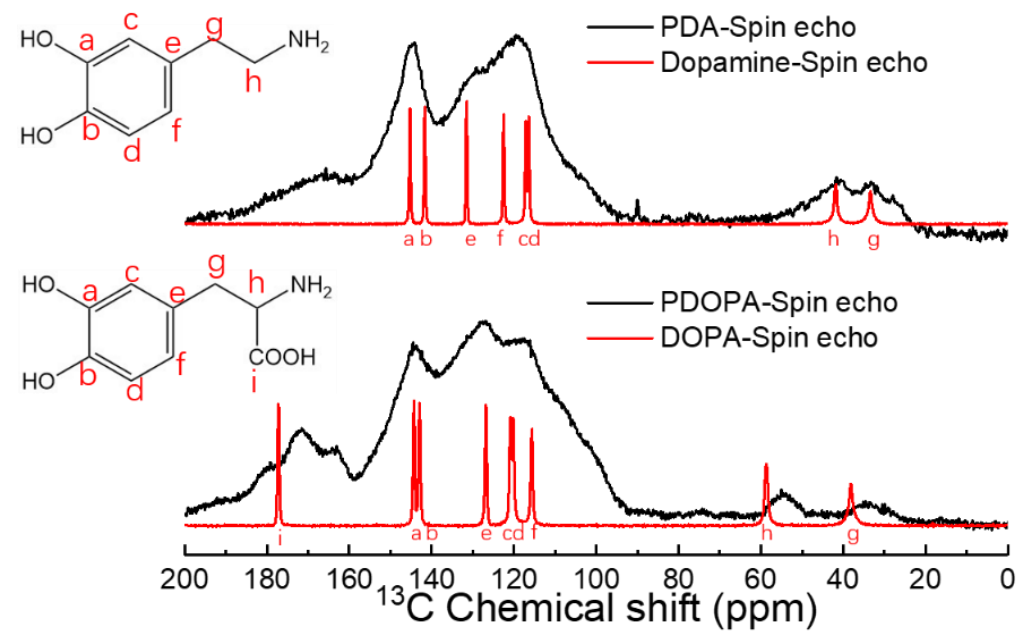

Figure S2. Spin echo spectra of PDA and PDOPA, and their monomers dopamine and L-DOPA (DOPA). The peaks denoted with a to i have corresponding carbons in proposed chemical structures.

(a)

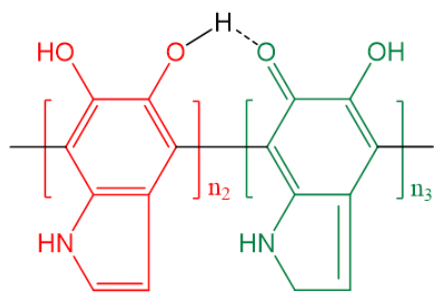

(b)

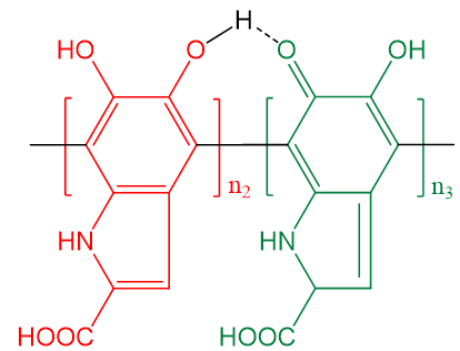

Figure S3. Hydrogen bonds between $\mathrm{C}-\mathrm{O}-\mathrm{H}$ group in $\mathrm{I} / \mathrm{I}^{\prime}$ and $\mathrm{C}=\mathrm{O}$ group in $\mathrm{II} / \mathrm{II}$ ' in a single chain 
of (a) PDA or (b) PDOPA.

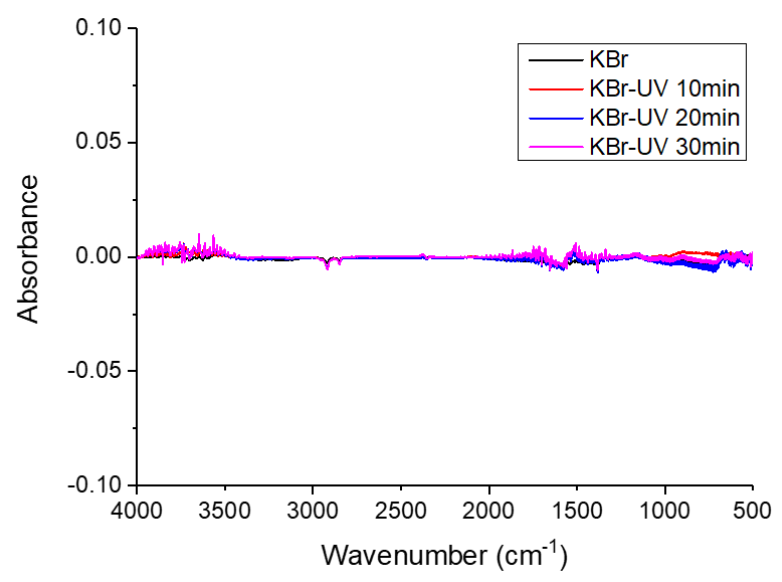

Figure S4. FTIR spectrum of potassium bromide $(\mathrm{KBr})$ pellet. After UV radiation, no $\mathrm{CO}_{2}$ peak $\left(2338 \mathrm{~cm}^{-1}\right)$ appears.

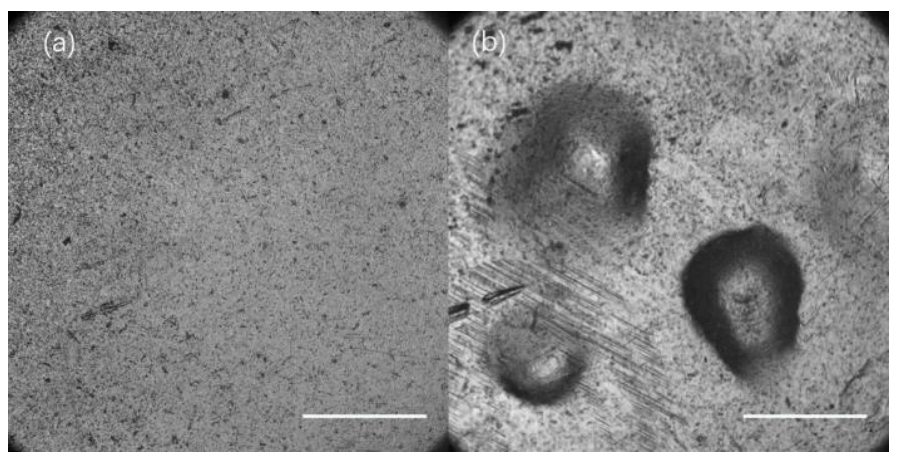

Figure S5. (a) Optical microscopy image of eumelanin- $\mathrm{KBr}$ pellet before UV exposure. (b) Optical microscopy image of eumelanin-KBr pellet after $30 \mathrm{~min}$ UV exposure. Scale bar: $1 \mathrm{~mm}$.

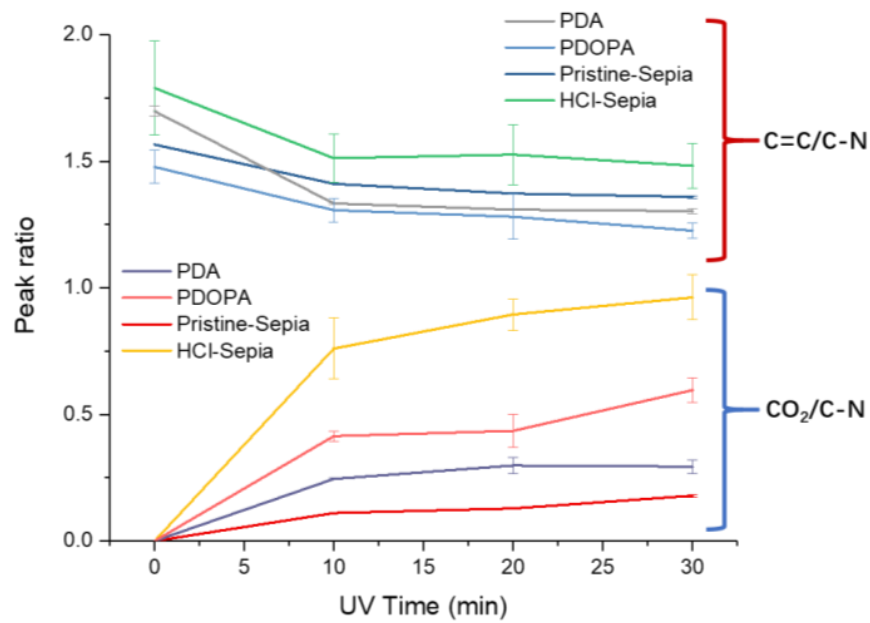

Figure S6. Absorbance change curves of the $\mathrm{C}=\mathrm{C}$ stretching and $\mathrm{CO}_{2}$ corresponding to the $\mathrm{C}-\mathrm{N}$ peak at $1290 \mathrm{~cm}^{-1}$ (PDA and PDOPA) and $1263 \mathrm{~cm}^{-1}$ (HCl-sepia eumelanin), or the peak centered at $1378 \mathrm{~cm}^{-1}$ (Sepia eumelanin). 
<smiles>CC(O)c1cc[nH]c1C(C)O</smiles><smiles>CC(=O)c1cc[nH]c1C(C)=O</smiles>

Figure S7. Other candidates for final products. (a) Example unit with carboxyl groups. (b) Example unit with carbonyl groups in ketone structure.

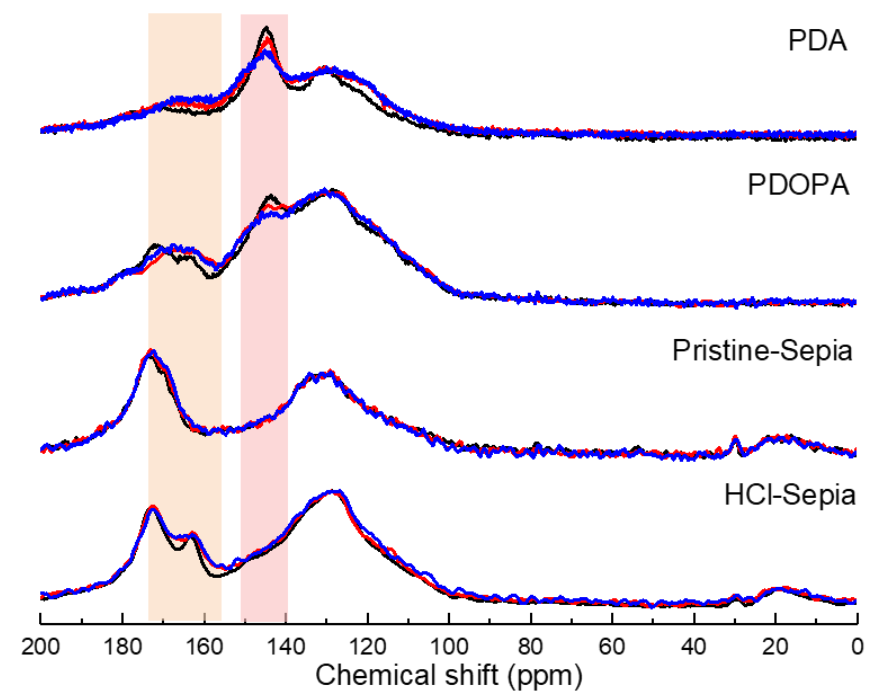

Figure S8. Dipolar dephasing spectra of four types of eumelanins before (black) and after UVA expo-sure for 1 (red) or 2 hours (blue).

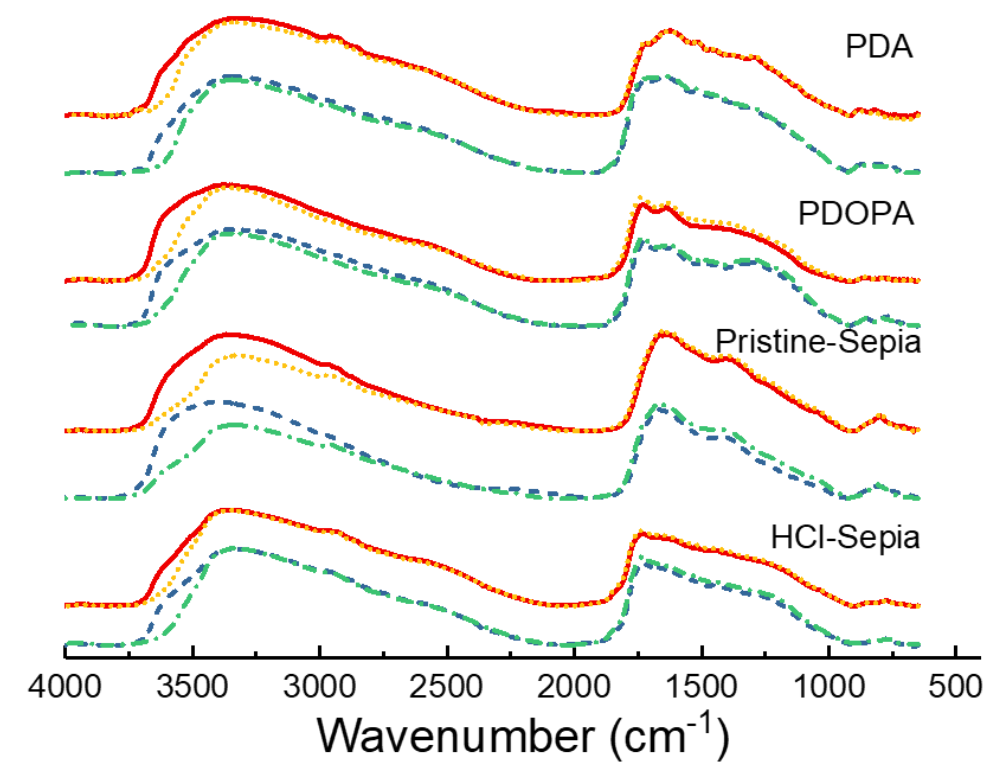

Figure S9. The full DRIFTS spectra of both original (red solid) and UVA-treated (blue dash) eumelanin powders, and their spectra (yellow dot and green dash-dot) after removing moisture (heating under Argon ventilation). 